\title{
Entropy in the early universe
}

\section{Llorenç Espinosa-Portalés ${ }^{a, *}$}

${ }^{a}$ Instituto de Física Teórica, Universidad Autónoma de Madrid Cantoblanco, 28049 Madrid, Spain

E-mail: 1lorenc.espinosa@uam.es

Most of the evolution of the universe takes place in thermal equilibrium, i.e. its dynamics is reversible. This can be directly deduced from the Friedmann equations, which are invariant under time inversion. Nevertheless, during some events the universe is certainly out of equilibrium and, thus, its evolution becomes irreversible. Such irreversibility can be incorporated into General relativity by means of a covariant variational description. When applied to cosmology, this delivers a modification of the second Friedmann equations that predicts an acceleration of the expansion during periods of entropy production. In light of this, we investigate how the growth of entropy of the causal horizon in an open inflation scenario may explain the current accelerated expansion of the universe without the need of a cosmological constant.

*** The European Physical Society Conference on High Energy Physics (EPS-HEP2021), ***

*** 26-30 July $2021 * * *$

*** Online conference, jointly organized by Universität Hamburg and the research center DESY ***

${ }^{*}$ Speaker 


\section{Introduction}

General Relativity, formulated more than a century ago, provides a successful description of all observed gravitational phenomena. Nevertheless, it is a classical theory and lacks a UV-complete quantum counterpart. The thermodynamic behavior of black holes points towards the existence of yet unknown microphysical quantum degrees of freedom. Hence, entropy seems to be part of the bridge between classical and quantum gravity.

Motivated by the relevance of thermodynamics in gravity, we review the variational formalism of non-equilibrium thermodynamics in General Relativity developed in [1]. This shows the need for a modification of the Einstein field equations and the emergence of entropic forces due to the tendency of any physical system, also gravitational, to increase its entropy.

This result is of particular relevance in Friedmann-Lemaitre-Robertson-Walker (FLRW) cosmology, where it delivers a modification of the second Friedmann equation. This predicts the appearance of an accelerating force when the universe departs from equilibrium in compliance with the laws of thermodynamics. These departures from equilibrium are usually expected to be short-lived. However, the increase in entropy associated to the causal horizon of the universe is sustained. We review how it may generate an acceleration consistent with current observations as explored in [2].

\section{Reversibility in Cosmology}

Let us review the basics of a homogeneous and isotropic universe, which is described by the FLRW metric and characterized by a scale factor $a(t)$ and a curvature parameter $k$. This space-time is filled with a perfect fluid, described by a stress-energy tensor $T^{\mu \nu}$, which is a function the density $\rho$ and the pressure $p$. The Einstein field equations for this metric and matter content deliver the dynamics for the scale factor, the well-known Friedmann equations

$$
H^{2}+\frac{k}{a^{2}}=\frac{8 \pi G}{3} \rho \quad \frac{\ddot{a}}{a}=-\frac{4 \pi G}{3}(\rho+3 p),
$$

where $H=\dot{a} / a$ is the Hubble parameter. There is a constraint on the stress-energy tensor due to the Einstein field equations and the Bianchi identities, namely its covariant conservation $D_{\mu} T^{\mu \nu}=0$. From this constraint one can derive the continuity equation

$$
\dot{\rho}+3 H(\rho+p)=0 .
$$

However, one can also derive this equation from the second law of thermodynamics. Indeed, changes in entropy are related to changes in internal energy and work $T d S=\delta U+\delta W$. If we apply this to a region of fixed comoving volume $a(t)^{3}$ we get

$$
T \frac{d S}{d t}=\frac{d}{d t}\left(\rho a^{3}\right)+p \frac{d}{d t}\left(a^{3}\right) .
$$

If the expansion of the universe is reversible, we can set the LHS to 0 and recover the continuity equation. However, this is only true in thermodynamical equilibrium and, in general, entropy is a monotonically increasing function of time. Most of the expansion history of the universe is 
indeed adiabatic. However, it is out-of-equilibrium at certain key points such as (p)reheating, phase transitions or gravitational collapse. Allowing for a time-varying entropy implies the addition of a term in the continuity equation

$$
\dot{\rho}+3 H(\rho+p)=\frac{T \dot{S}}{a^{3}} .
$$

Combining this with the first Friedmann equation we obtain a modified, non-equilibrium second Friedmann equation

$$
\frac{\ddot{a}}{a}=-\frac{4 \pi G}{3}\left(\rho+3 p-\frac{T \dot{S}}{a^{3} H}\right) .
$$

In principle, this evolution equation does not seem to be compatible with the Einstein field equations. In order to achieve that, the laws of thermodynamics need to be rigorously incorporated into the computation of the equations of motion. This can be achieved by applying to General Relativity the variational formalism of non-equilibrium thermodynamics, developed in another context by Gay-Balmaz and Yoshimura [3, 4].

\section{Entropic forces in General Relativity}

Entropic forces arise in any physical system as a result of its tendency to increase entropy, in compliance with the second law of thermodynamics. Let us consider the Einstein-Hilbert action and a matter term, which may have a dependency on the entropy density

$$
\mathcal{S}=\frac{1}{2 \kappa} \int d^{4} x \sqrt{-g} R+\int d^{4} x \mathcal{L}_{m}\left(g_{\mu \nu}, s\right)
$$

where $\kappa=8 \pi G$ is the gravitational coupling and $R$ is the Ricci scalar. The dependency on the entropy density $s$ requires treating it as an additional degree of freedom at the action level. It encodes additional physics whose detailed description is disregarded. The action principle states then that the equations of motion are obtained by solving the equation $\delta \mathcal{S}=0$

Nevertheless, entropy is not a free dynamical variable. On the contrary, its evolution is dictated by the second law of thermodynamics. Therefore, it is necessary to constrain the variational problem in order to enforce the second law of thermodynamics. This is achieved by imposing a variational constraint [3, 4]. In General Relativity it takes the form [1]

$$
\frac{\partial \mathcal{L}_{m}}{\partial s} \delta s=\frac{1}{2} \sqrt{-g} f_{\mu \nu} \delta g^{\mu \nu},
$$

where $f_{\mu \nu}$ is the friction or entropic force tensor. The solution to the constrained variational problem is then provided by the non-equilibrium Einstein field equations

$$
R_{\mu \nu}-\frac{1}{2} R g_{\mu \nu}=\kappa\left(T_{\mu v}-f_{\mu \nu}\right) .
$$

It includes the standard graviational (geometric) and matter terms plus an entropic correction, which shows the relevance of non-equilibrium thermodynamics in General Relativity. Note that the Bianchi identities allow for the covariant non-conservation of the stress-energy tensor, i.e. $D_{\mu} T^{\mu v}=D_{\mu} f^{\mu \nu}$. 
The precise meaning of the entropic force tensor arising from the variational constraint is unclear. It needs to be supplemented with the phenomenological constraint, an equation that relates time evolution of the entropy with that of the physical degrees of freedom.

One can obtain a proper notion of time evolution in the Hamiltonian formulation of General Relativity o Arnowitt-Deser-Misner (ADM) formalism. Space-time is foliated into constant-time hypersurfaces with normal vector $n$. Then the space-time metric is split into components perpendicular and parallel to the hypersurfaces $g_{\mu \nu}=-n_{\mu} n_{\nu}+h_{\mu \nu}$. The only physical degrees of freedom of the 4-metric $g_{\mu \nu}$ are encoded into the 3-metric $h_{\mu \nu}$, which has a time evolution along the normal vector to the hypersurfaces. The values of $h_{\mu \nu}$ and $\dot{h}_{\mu \nu}$ at a given hypersurface pose a well-defined initial value problem. In this setup one can argue the phenomenological constraint to be [1]

$$
\frac{\partial \mathcal{L}}{\partial s} £_{n} s=\frac{1}{2} N \sqrt{h} \tilde{f}_{i j} £_{n} h^{i j},
$$

where $\mathfrak{f}_{n}$ is the Lie derivative along $n$ and $\tilde{f}_{i j}$ are the spatial components of the projection of $f_{\mu \nu}$ onto the hypersurface. We find then a consistent and first-principles way to integrate the second law of thermodynamics in a variational formulation of General Relativity.

\section{Cosmic acceleration from entropic forces}

The variational formulation of entropic forces in General Relativity can be applied to a cosmological set-up. Assuming a FLRW ansatz for the metric and a homogeneous and isotropic fluid, one finds a modification of the second Friedmann equation as in eq. 5. This means that the result obtained by a heuristic application of the second law of thermodynamics can also be justified in a rigorous way.

Since $\dot{S}>0$ by the second law of thermodynamics and $H>0$ in an expanding universe, one is lead to the conclusion that out-of-equilibrium processes tend to accelerate the expansion of the universe. Even though most of the expansion history of the universe is adiabatic, one can think of non-equilibrium processes such as (p)reheating, phase transitions or gravitational collapse. These events may cause a short-term acceleration of the expansion. It is our opinion that they should be revisited in light of our results.

Indeed the universe is currently experiencing an accelerated expansion of unknown origin. The introduction of a cosmological constant into the Einstein-Hilbert action is currently the accepted model, forming with cold dark matter the $\Lambda$ CDM paradigm. Extensions of it in form of modified gravity or dark energy have been considered, but none seems to be competitive so far.

Instead of modifying gravity or adding an additional, dark energy content, we suggest the increase in entropy of the causal horizon of the universe to be responsible for the accelerated expansion. The entropy of a horizon $\mathcal{H}$ can be taken into account in the action by means of a Gibbons-Hawking-York (GHY) term

$$
S_{G H Y}=\frac{1}{\kappa} \int_{\mathcal{H}} d^{3} y \sqrt{h} K
$$

where $h$ is the determinant of the 3-metric of the horizon and $K$ is the trace of its extrinsic curvature. This term can be reinterpreted as a contribution to the thermodynamic internal energy of the form

$$
S_{G H Y}=-\int d t T_{H} S_{H},
$$


where $T_{H}$ and $S_{H}$ are, respectively, the temperature and entropy assigned to the horizon. If localized, this action describes a thermodynamic object. If extended, such as the causal horizon of the universe, it introduces an effective entropic fluid.

We consider an open universe nucleated in de-Sitter space-time [5]. If the subsequent inflationary period inside the true vacuum bubble lasts for enough e-folds $(N \geq 70)$, the local metric is essentially flat, although the bubble walls are still at a finite coordinate distance. This is the true causal horizon of the universe in this scenario, which induces an entropic fluid via GHY term with energy density

$$
\rho_{H} a^{2}=\frac{T_{H} S_{H}}{a}=\frac{x_{0}}{2 G} \sinh \left(2 a_{0} H_{0} \eta\right), \quad x_{0} \equiv \frac{1-\Omega_{0}}{\Omega_{0}}=e^{-2 N}\left(\frac{T_{\mathrm{rh}}}{T_{\mathrm{eq}}}\right)^{2}\left(1+z_{\mathrm{eq}}\right) .
$$

where $\eta$ is the conformal time, $\Omega_{0}$ is the density parameter, $T_{\mathrm{rh}}$ is the reheating temperature, $T_{\mathrm{eq}}$ and $z_{\mathrm{eq}}$ are, respectively, the temperature and redshift at matter-radiation equality. Introducing $\tau=a_{0} H_{0} \eta$ one can write the second Friedmann equation in conformal time as

$$
\left(\frac{a^{\prime}}{a_{0}}\right)^{2}=\Omega_{M}\left(\frac{a}{a_{0}}\right)+\Omega_{K}\left(\frac{a}{a_{0}}\right)^{2}+\frac{4 \pi}{3} \Omega_{K}\left(\frac{a}{a_{0}}\right)^{2} \sinh (2 \tau),
$$

where $\Omega_{M}$ is the matter density paremeter and $\Omega_{K}$ is the curvature parameter. We call this the general relatvistic entropic acceleration (GREA) theory.

The solution of this equation with cosmological parameters consistent with the CMB values (Planck 2018: $\Omega_{M} \simeq 0.31, \Omega_{K} \simeq 0.0006, h_{0} \simeq 0.68$ ) and initial conditions deep in the matter era, $a_{i}(\tau)=a_{0} \Omega_{M} \tau^{2} / 4$ gives generic accelerating behaviour beyond the scale factor $a \sim 1 / 2$ (i.e. $z \sim$ 1 ), see fig 1 . This is consistent with the current observed acceleration of the universe and may even resolve the Hubble tension [6]: it provides a way to obtain a present value of $H_{0}$ from the CMB that is in agreement with late-universe observations.
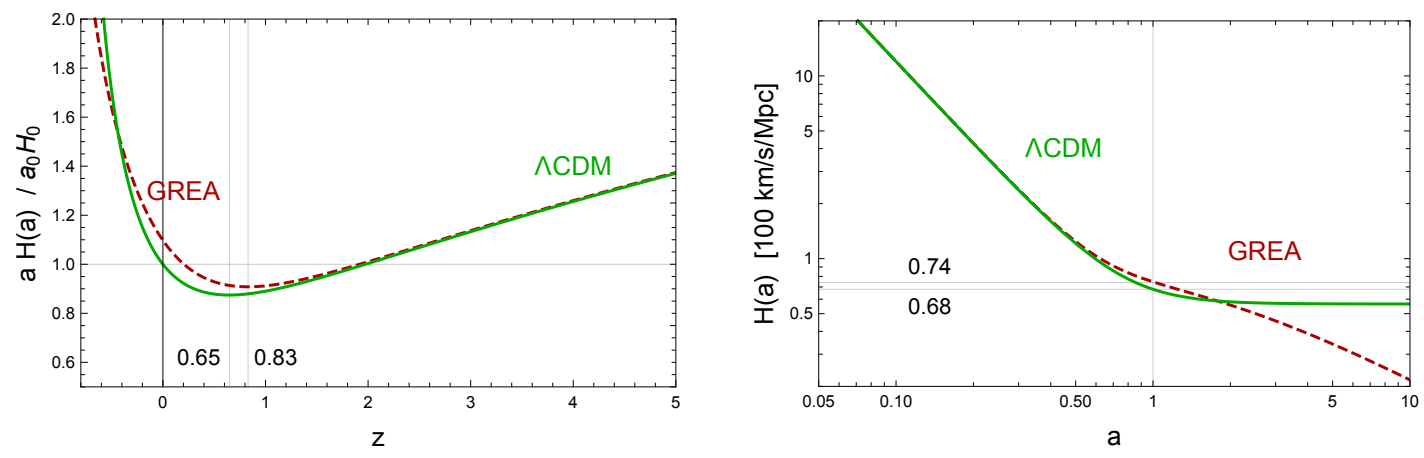

Figure 1: The left plot shows the evolution of the inverse comoving horizon with the coasting point for each model, at $z \simeq 0.65$ for $\Lambda$ CDM (in green) and $z \simeq 0.83$ for GREA (in red). The right plot shows the evolution of the rate of expansion. For GREA the present rate of expansion is approximately $74 \mathrm{~km} / \mathrm{s} / \mathrm{Mpc}$, compared with the value of $68 \mathrm{~km} / \mathrm{s} / \mathrm{Mpc}$ predicted by $\Lambda \mathrm{CDM}$, in agreement with the asymptotic value at the CMB.

\section{Conclusions}

Non-equilibrium phenomena may have a deep impact in our understanding of certain events in the expansion history of the universe. A heuristic modification of the continuity equation of the 
cosmic fluid, in compliance with the second law of thermodynamics, implies a modification of the second Friedmann equation. Their rigurous inclusion within General Relativity requires adding a constraint to the variational problem of the Einstein-Hilbert action. We find that the solution to this constrained problem delivers an entropic modification to the Einstein field equations. This confirms the need for modifying the second Friedmann equation and, thus, the dynamics of the expansion of the universe when entropy is not conserved, but rather increases in time. According to this modification, entropy-producing processes, such as (p)reheating, phase transitions or gravitational collapse, may lead to a short-term acceleration of the expansion of the universe. We plan to explore the impact of such short acceleration phases in the future.

The entropy assigned to horizons also plays a role in the dynamics of space-time. Their contribution to the action via a GHY term can bee interpreted in thermodynamic terms and source an effective entropic fluid. When applied to an almost flat universe arising in the open inflation scenario, the increase in entropy of its causal horizon may explain the current accelerated expansion of the universe, as well as the tension between the value of $H_{0}$ obtained from early- and late-universe observations. Future research will determine the full viability of this theory.

\section{Acknowledgments}

I thank Juan García-Bellido for a fruitful collaboration that led to the results reviewed here. This work is supported by the Spanish Research Project PGC2018-094773-B-C32 (MINECOFEDER), the Centro de Excelencia Severo Ochoa Program SEV-2016-0597 and a fellowship from "La Caixa" Foundation (ID 100010434) with fellowship code LCF/BQ/IN18/11660041 and the European Union Horizon 2020 research and innovation programme under the Marie SklodowskaCurie grant agreement No. 713673.

\section{References}

[1] L. Espinosa-Portales and J. Garcia-Bellido, Covariant formulation of non-equilibrium thermodynamics in General Relativity, Physics of the Dark Universe 34 (2021) 100893 [2106. 16012].

[2] J. Garcia-Bellido and L. Espinosa-Portales, Cosmic acceleration from first principles, Physics of the Dark Universe 34 (2021) 100892 [2106. 16014].

[3] F. Gay-Balmaz and H. Yoshimura, A lagrangian variational formulation for nonequilibrium thermodynamics. part i: Discrete systems, Journal of Geometry and Physics 111 (2017) 169.

[4] F. Gay-Balmaz and H. Yoshimura, A lagrangian variational formulation for nonequilibrium thermodynamics. part ii: Continuum systems, Journal of Geometry and Physics 111 (2017) 194.

[5] A.D. Linde, M. Sasaki and T. Tanaka, CMB in open inflation, Phys. Rev. D59 (1999) 123522 [astro-ph/9901135].

[6] A.G. Riess, The Expansion of the Universe is Faster than Expected, Nature Rev. Phys. 2 (2019) 10 [2001.03624]. 